\title{
Effects of the GABA-uptake inhibitor tiagabine on electroencephalogram, spike-wave discharges and behaviour of rats
}

\author{
A.M.L. Coenen ${ }^{*}$, E.H.M. Blezer, E.L.J.M. van Luijtelaar \\ NICI, Department of Psychology, University of Nijmegen, Nijmegen, the Netherlands
}

Received 19 December 1994; revised 21 February 1995; accepted 22 February 1995

\begin{abstract}
Effects of the anticonvulsant tiagabine in doses of 1,3 and $10 \mathrm{mg} / \mathrm{kg}$ were investigated on electroencephalogram (EEG), spike-wave discharges and behaviour of WAG/Rij rats. These rats are considered as an animal model of generalized, non-convulsive, absence epilepsy. WAG/Rij rats spontaneously show a considerable number of spike-wave discharges in their EEG. These discharges can be facilitated by GABA agonists. The facilitatory effects of these agonists are completely opposite to their effects on convulsive seizures, which are reduced by these drugs. Tiagabine enhances the effects on the GABA system, since it acts as a GABA re-uptake inhibitor. According to expectations, tiagabine enhanced in a dose-related way both the number and mean duration of spike-wave discharges. The low dose of $1 \mathrm{mg} / \mathrm{kg}$ had almost no effects, but doses of 3 and $10 \mathrm{mg} / \mathrm{kg}$ were effective. Furthermore, tiagabine in the latter two doses increased the power in the higher beta band of the background EEG, whereas no significant changes in behavioural parameters were found. An unexpected finding was the occurrence of a second type of spike-wave discharges. These were again seen with the two higher doses of tiagabine, while $1 \mathrm{mg} / \mathrm{kg}$ had no effect. An assumption is that this second type of discharges are forerunners of genuine spike-wave discharges. In general, this experiment supports that non-convulsive epilepsy is associated with a GABA hyperfunction. It also underlines the biochemical differences of convulsive and non-convulsive animal models of epilepsy. Tiagabine, with its GABA-mimetic properties, belongs to the category of drugs effective in convulsive animal models and not in non-convulsive models of epilepsy.
\end{abstract}

Keywords: Tiagabine; GABA-uptake inhibitor; Spike-wave discharges; Electroencephalogram; Spectral analysis; Behaviour; Rats

\section{Introduction}

Tiagabine is a recertly developed potent and centrally acting selective GABA-uptake inhibitor. Its

\footnotetext{
* Corresponding author. NICI, Department of Psychology, University of Nijmegen, P.O. Box 9104, $6500 \mathrm{HE} \mathrm{Nijmegen,} \mathrm{The}$ Netherlands. Tel.: +31.80.612545; Fax: +31.80 .616066 , E-mail COENEN@NICI.KUN.NL.
}

anticonvulsant activity is established in several models of epilepsy and its anticonvulsant action is already effective in doses which do not produce sedation or motoric side-effects [9]. Tiagabine inhibits the re-uptake of GABA in synaptic terminals, implicating that it enhances GABA-ergic functions in an indirect way [6]. It is generally agreed that GABA agonists, such as muscimol, reduce the number of seizures in models of convulsive epilepsy and that 
GABA antagonists, such as bicuculline and picrotoxine, enhance this type of epilepsy $[8,11]$. Interestingly, GABA plays an opposite role in models of non-convulsive epilepsy. In rats, spike-wave discharges of the absence type are facilitated by agonists and reduced by antagonists $[7,12,13,16]$. The pharmacological difference with respect to the role of GABA in convulsive and non-convulsive animal models of epilepsy is discussed in a review paper by Coenen et al. [5].

In the present experiment the GABA-uptake inhibitor tiagabine is investigated in a rat model for non-convulsive epilepsy, the WAG/Rij strain of rats. These rats show numerous spike-wave discharges of the non-convulsive type and are considered as an animal model for generalized absence epilepsy [5,15].

\section{Materials and methods}

Thirty-two male WAG/Rij rats were included in the experiment. All animals were seven months old and weighed 280-335 grams. Rats were chronically implanted with a tripolar EEG electrode set (Plastics One MS 333/2A) over the frontal and occipital cortex under deep pentobarbital anesthesia. The animals were allowed to recover for at least one week after surgery. Tap water and food pellets were always available.

One day prior to the experimental day, the animals were placed in a recording cage $(25 \mathrm{~cm} \times 35$ $\mathrm{cm} \times 30 \mathrm{~cm}$ ) and connected to recording leads for habituation and acquaintance. On the experimental day, EEG leads were connected to the polygraph and recordings started. Signals were amplified and filtered, with the high-pass filter set at $0.15 \mathrm{~Hz}$ and the low-pass filter at $70 \mathrm{~Hz}$. Signals were sampled at 180 $\mathrm{Hz}$ and digitized with a Codas system on a PC (Dataq Instruments). EEG data were stored on an optical disk for analysis. The EEG was recorded for one hour before and for two hours following drug or saline administration. Tiagabine was dissolved in saline and intraperitoneally injected in a volume of 1 $\mathrm{ml} / \mathrm{kg}$. Animals were divided into four groups $(n=$ 8): a control group injected with saline, a group receiving $1 \mathrm{mg} / \mathrm{kg}$ tiagabine, a group receiving 3 $\mathrm{mg} / \mathrm{kg}$ tiagabine and a group receiving $10 \mathrm{mg} / \mathrm{kg}$ tiagabine.
Behavioural observation took place for a period of 30 min starting immediately after drug administration. The observer was experienced in recording animal behaviour, but was not blind to the animal's condition. Behavioural data were stored on a portable Tandy computer and analysed with The Observer [10]. Periods of passive wakefulness were on-line recorded for a period of one hour directly after drug administration. The spectral content of the EEG during these periods of passive wakefulness was off-line established using a FFT routine (Dataq Instruments) and averaged for each animal [3]. The number and duration of spike-wave discharges in the EEG were separately determined in periods of $15 \mathrm{~min}$. Amplitude and frequency criteria for spike-wave discharges are elaborated previously [15]. In short, a spike-wave discharge was identified as such if it included a train of sharp spikes and slow waves with a frequency between 7 and $11 \mathrm{~Hz}$, whereas the minimum duration of the train was one second.

All EEG and behavioural data were analysed with a one-way analysis of variance with dose as factor (four levels). If appropriate, Duncan's post-hoc tests $(P<0.05)$ were used to evaluate differences between groups.

\section{Results}

\subsection{Effects of tiagabine on spike-wave discharges}

Tiagabine appeared to have potent facilitating effects on the number of spike-wave discharges (SWDs) and a clear dose-response relationship could be established. Effects of tiagabine on the number of SWDs in the cortical EEG are shown in Fig. 1. The group with the highest dose of tiagabine $(10 \mathrm{mg} / \mathrm{kg})$ has the greatest increase in the number of SWDs, whereas the group with the lowest dose $(1 \mathrm{mg} / \mathrm{kg})$ showed only a non-significant marginal increase. An analysis of variance showed significant differences between the four groups in the first $(F(3,28)=7.60$, $P<0.001)$, the second $(F(3,28)=14.70, \quad P<$ $0.0005)$, the third $(F(3,28)=9.47, P<0.0005)$, the fourth $(F(3,28)=4.57, \quad P<0.01)$ and the fifth $(F(3,28)=5.92, P<0.005) 15-\mathrm{min}$ period after drug administration. In all these 15 -min periods the num- 


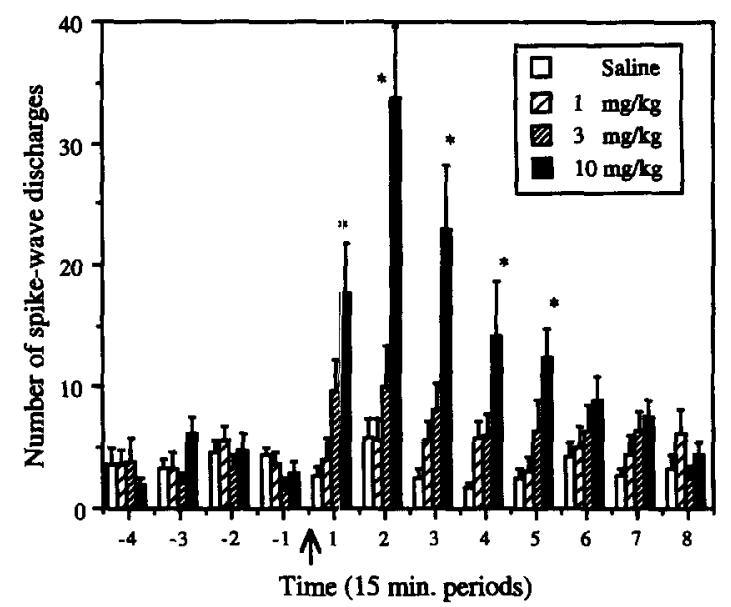

Fig. 1. Numbers of spike-wave discharges with SEMs per period of $15 \mathrm{~min}$, before and after liagabine administration. The arrow indicates injection of the drug. $*: P<0.05$.

ber of SWDs was higher for the $10 \mathrm{mg} / \mathrm{kg}$ group compared to the three other groups.

Due to tiagabine administration, the SWDs also increased in duration and a dose-response relationship existed for this increase in duration. The effects of different doses of tiagabine on the mean duration of SWDs are shown in Fig. 2. The effects of tiagabine on the duration last slightly longer than on the number of SWDs. Anallysis of variance showed significant differences between the four groups in the

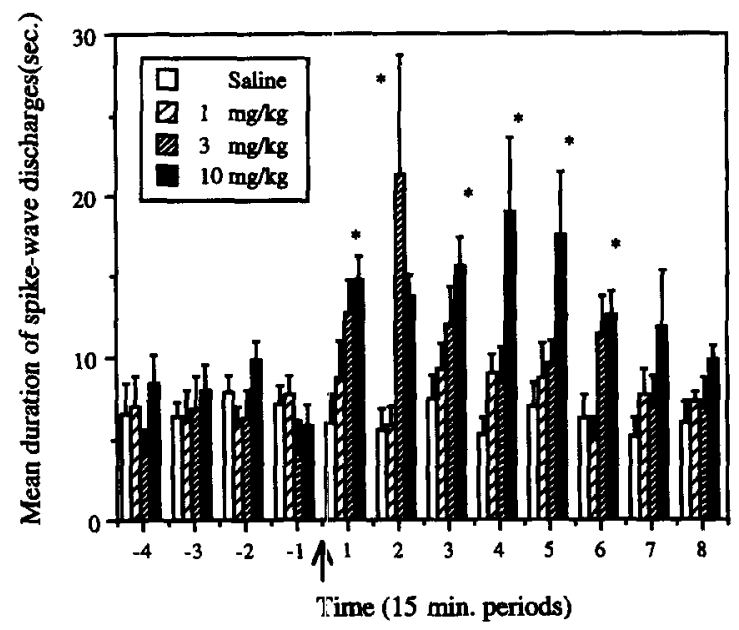

Fig. 2. Mean durations of single trains of spike-wave discharges with SEMs, before and after tiagabine administration. The arrow indicates injection of the drug. $*: P<0.05$. first $(F(3,28)=4,49, \quad P<0.05)$, the second $(F(3,28)=3.94, P<0.05)$, the third $(F(3,28)=$ $3.71, P<0.05)$, the fourth $(F(3,28)=4.97, P<$ $0.01)$, the fifth $(F(3,28)=3.69, P<0.05)$ and the sixth $(F(3,28)=4.70, P<0.01) 15-$ min period after drug administration. Generally, the highest dose group showed a prolonged duration compared to the lowest dose and the saline group.

\subsection{Effects of tiagabine on behaviour}

Behaviour was divided into three categories: exploratory or voluntary behaviour (walking, rearing and sniffing), automatic behaviour (eating, drinking and grooming) and immobile or passive behaviour [3]. There were no significant dose effects on the total time and mean durations that the animals were engaged in each of the behavioural categories. A marginal significant dose effect was found on the total time of exploratory behaviour $(F(3,28)=2.71$, $P<0.07$ ), but only the highest dose of tiagabine showed a decrease.

\subsection{Effects of tiagabine on the EEG spectrum}

Effects of tiagabine on the spectral content of the EEG were established on the EEG accompanying passive wakefulness when the animal shows immobile behaviour. Spectral analysis was based on time periods of minimally $60 \mathrm{~s}$. In general, it appeared that tiagabine had little effects on the spectral EEG content. Only a dose effect in the beta band of 25 to $39 \mathrm{~Hz}$ was found: the four groups differed in the power of this frequency band $(F(3,27)=4.10, P<$ 0.05 ). Animals with doses of 3 and $10 \mathrm{mg} / \mathrm{kg}$ tiagabine showed more power in this band compared to control animals and to rats with $1 \mathrm{mg} / \mathrm{kg}$ tiagabine. Furthermore, animals with a dose of 3 $\mathrm{mg} / \mathrm{kg}$ had more power in this band than the animals with a dose of $1 \mathrm{mg} / \mathrm{kg}$ tiagabine.

\subsection{Other EEG effects of tiagabine}

Besides the effects on SWDs and the spectral content of the EEG, tiagabine had another striking effect on the EEG. After administration of the two highest doses of tiagabine ( 3 and $10 \mathrm{mg} / \mathrm{kg}$ ), a 


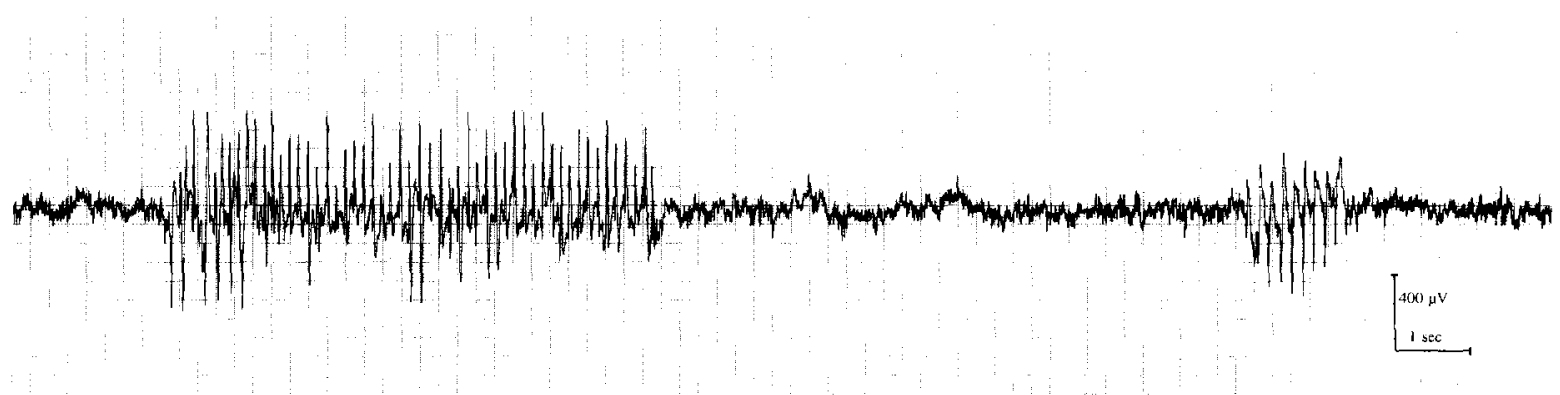

Fig. 3. A cortical EEG recording containing a common type I spike-wave discharge (left-middle), as well as a type II spike-wave discharge (right). Type II discharges are mostly short-lasting and spikes have an opposite polarity as the normal type I discharges. Time and voltage are calibrated.

phenomenon occurred which we originally described as type II SWDs [15]. These are short-lasting SWDs with a deviant morphology, not having a behavioural concomitant. In Fig. 3 an example of such a phenomenon is shown, together with a regular type I spike-wave discharge. No such type II phenomena were observed in baseline conditions, while rats in the 0 and $1 \mathrm{mg} / \mathrm{kg}$ groups also did not show any of these type II spike-wave discharges. They became visible during the first 15 -min period after administration of 3 and $10 \mathrm{mg} / \mathrm{kg}$ tiagabine, and increased in number during the second and third 15-min recording period. During the following 15 -min peri-

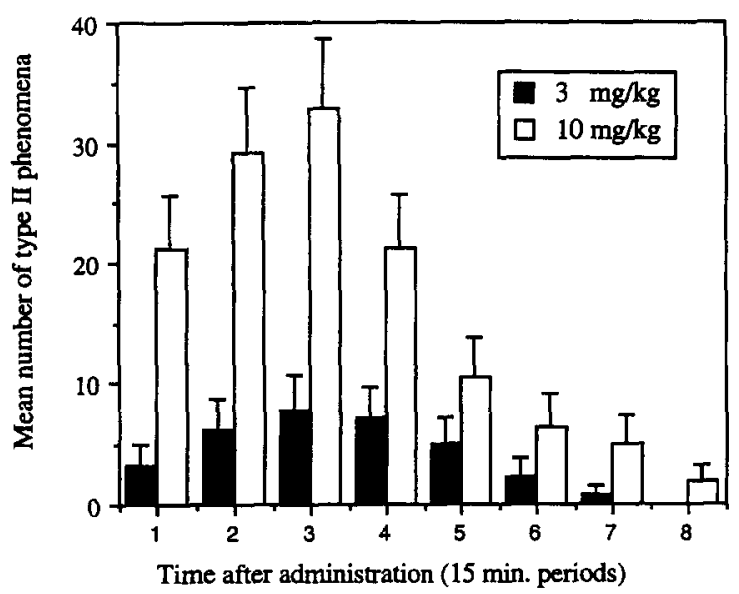

Fig. 4. Number of type II spike-wave discharges with SEMs occurring after administration of 3 and $10 \mathrm{mg} / \mathrm{kg}$ tiagabine. No such phenomena occur after administration of 0 and $1 \mathrm{mg} / \mathrm{kg}$ tiagabine. Results are presented in periods of $15 \mathrm{~min}$ after drug administration. ods the number of the phenomena slowly decreased reaching a zero base-line value in the eighth 15 -min period. During all periods the number of type II phenomena is greater for $10 \mathrm{mg} / \mathrm{kg}$ animals compared to $3 \mathrm{mg} / \mathrm{kg}$ animals, which suggests a doseresponse relationship. Mean numbers of type II phenomena for the 3 and $10 \mathrm{mg} / \mathrm{kg}$ tiagabine groups are shown in Fig. 4.

\section{Discussion}

Tiagabine had effects on the number as well as on the duration of SWDs and both parameters increased as a result of drug administration. The lowest dose of $1 \mathrm{mg} / \mathrm{kg}$ had still small effects while doses of 3 and $10 \mathrm{mg} / \mathrm{kg}$ tiagabine show considerable actions. In general, little effects were seen on the spectral characteristics of the EEG. Merely the power in the beta range increased after tiagabine with only the two highest doses. No corresponding behavioural signs such as an increase in alertness or exploratory behaviour were found. Moreover, exploratory behaviour had even a slight tendency to decrease. The finding of an increase in power of the beta band of the EEG, which is not associated with parallel effects on behaviour such as an increase in active behaviour, seems contradictory. It is known, however, that certain types of drugs produce still larger dissociating effects on EEG and behaviour. With benzodiazepines, for example, even hypnotic and sedatory actions occur simultaneously with an increase in the power of the beta band [3]. This is known as pharma- 
cological dissociation: a drug may have opposite effects on EEG and behaviour [4].

Next to the facilitation of SWDs, an unexpected effect of tiagabine was found: the appearance of another type of paroxysmal activity. These phenomena, also called type II SWDs, were earlier described as spontaneously occurring in WAG/Rij rats [15]. In the present experiment, they were clearly seen after administration of the two highest doses of tiagabine of 3 and $10 \mathrm{mg} / \mathrm{kg}$. The dose of $1 \mathrm{mg} / \mathrm{kg}$ tiagabine did not produce these phenomena. From occasional and cortical mapping observations, it appeared that these type II phenomena are bilateral synchronous, but have a more local, occipital origin than the commonly found type I SWDs, which seem to be more generalized over the frontal cortex [14]. The occurrence of these still enigmatic type II paroxysms seems to be a process in line with the enhancement of the more generally occurring type I SWDs. An assumption is that these type II phenomena might be forerunners of genuine type I SWDs. However, it is so far not described that muscimol, another GABAmimetic drug enhancing spike-wave discharges, also give rise to these type II SWDs. It might be that aggravation of type II SWDs is restricted to a specific mode of action of the drug on the GABA-ergic system.

Tiagabine is a drug which stimulates GABA-ergic functions in an indirect way, by inhibition of the re-uptake of GABA in synaptic terminals [9]. The facilitating effects on the non-convulsive type of epilepsy by GABA-stimulating drugs is already earlier described by us $[12,13]$, as well as by others $[7,16]$. Non-convulsive discharges react in an opposite way to GABA-ergic facilitation as convulsive or tonic-clonic seizures. The latter are strongly suppressed by GABA agonists and facilitated by antagonists. In general, this means that the results obtained with tiagabine fit into this overall picture. They again underline that GABA is involved in absence epilepsy. Stimulation of GABA causes an increase of spikewave activity, which suggests that non-convulsive epilepsy is associated with GABA-ergic hyperfunction. This implies that the mechanism of action is different from drugs which inhibit non-convulsive seizures, such as the benzodiazepines. Though the latter drugs are generally regarded as agonists at GABA receptors, the opposite results of the SWD- enhancing GABA agonists on one hand and the SWD-reducing benzodiazepines on the other [3] proves that the statement that benzodiazepines act as genuine GABA agonists is presumably not completely true.

The distinction between typical anti-convulsive drugs and anti-absence drugs becomes more and more obvious and indicates a biochemical difference in the regulation of systems underlying the two main types of epilepsy. There are more anticonvulsants such as diphenylhydantoin and carbamazepine, which also aggravate SWDs in WAG/Rij rats [12]. These drugs are also ineffective against absences in humans, but are effective in tonic-clonic seizures [1,2]. Regarding the effectiveness of tiagabine in several models for convulsive epilepsy and considering the results presented here, tiagabine seems to belong to the category of anti-convulsive drugs. Tiagabine has been shown to be efficacious as add-on treatment in complex partial seizures with and without secondary generalization (Richens et al., submitted). It should be worthwhile to investigate further the cognitive profile of tiagabine and to compare it with the profiles of other anti-convulsants. It is possible that the indirect mode of action on GABA might give rise to fewer adverse effects on behaviour and cognition than existing drugs directly modulating the GABA-ergic system or other currently used anticonvulsants such as diphenylhydantoin and carbamazepine. The choice of anti-convulsants is also partially based on the lack of cognitive side effects. In the present experiment, it is already shown that tiagabine showed almost no behavioural effects and only slight effects on the background EEG.

\section{Acknowledgements}

The authors are indebted to Dr. H. Mengel of Novo Nordisk, Bagsvaerd, Danmark, for kind donation of tiagabine.

\section{References}

[1] Bruni, J. and Albright, P.S., The clinical pharmacology of antiepileptic drugs, Clin. Neuropharmacol., 7 (1984) 1-34.

[2] Cereghino, J.J., Brock, J.T., van Meter, J.C., Penry, J.K., 
Smith, L.D. and White, B.G., Carbamazepin for epilepsy. A controlled prospective evaluation, Neurology, 24 (1974) 401-410.

[3] Coenen, A.M.L. and van Luijtelaar, E.L.J.M., Effects of diazepam and two beta-carbolines on epileptic activity and on EEG and behavior in rats with absence epilepsy, Pharmacol. Biochem. Behav., 32 (1989) 27-35.

[4] Coenen, A.M.L. and van Luijtelaar, E.L.J.M., Pharmacological dissociation of EEG and behavior: $A$ basic problem in sleep-wake classification, Sleep, 14 (1991) 464-465

[5] Coenen, A.M.L., Drinkenburg, W.H.I.M., Inoue, M. and van Luijtelaar, E.L.J.M., Genetic models of absence epilepsy, with emphasis on the WAG/Rij strain of rats, Epilepsy Res., 12 (1992) 75-86.

[6] Iversen, L.L. and Johnston, G.A.R., GABA-uptake in rat central nervous system; comparison of uptake in slices and homogenates and the effects of some inhibitors, J. Neurochem., 18 (1971) 1939

[7] Micheletti, G., Marescaux, C., Vergnes, M., Rumbach, L. and Warter, J.-M., Effects of the GABA mimics and GABA antagonists on spontaneous non-convulsive seizures in Wistar rats. In: G. Bartholini, L. Bossi, K.G. Lloyd and M.L. Morselli (Eds.), Epilepsy and GABA Receptor Agonists. Basic and Therapeutic Research, L.E.R.S. Monograph Series Vol. 3, Raven Press, New York, 1985, pp. 129-137.

[8] Morita, K., Okamoto, M., Seki, K. and Wada, J.A., Suppression of amygdala kindled seizures in cats by enhanced GABA-ergic transmission in the substantia innominata, Exp. Neurol., 89 (1985) 225-236.

[9] Nielsen, E.B., Suzdak, P.D., Andersen, K.E., Knutsen, L.J.S.,
Sonnewald, U. and Braestrup, C., Characterization of tiagabine (NO-328), a new and selective GABA uptake inhibitor, Eur. J. Pharmacol., 196 (1991) 257-266.

[10] Noldus, L.P.J.J., The observer: A software system for collection and analysis of observational data, Behav. Res. Meth. Instrum. Computers, 23 (1991) 415-429.

[11] Olsen, R.W., The GABA postsynaptic membrane receptorionophore complex site of action of convulsant and anticonvulsant drugs, Mol. Cell. Biochem., 39 (1981) 261-279.

[12] Peeters, B.W.M.M., Spooren, W.P.J.M., van Luijtelaar, E.L.J.M. and Coenen, A.M.L., The WAG/Rij model for absence epilepsy: Anticonvulsant drug evaluation, Neurosci. Res. Commun., 2 (1988) 93-97.

[13] Peeters, B.W.M.M., van Rijn, C.M., Vossen, J.M.H. and Coenen, A.M.L., Effects of GABAergic agents on spontaneous non-convulsive epilepsy, EEG and behaviour, in the WAG/Rij inbred strain of rats, Life Sci., 45 (1989) 11711176.

[14] Reuvers, R., Coenen, A. and van Luijtelaar, G., Properties of sleep spindles and other oscillations in the EEG of rats. In: A.M.L. Coenen (Ed.), Sleep-Wake Research in The Netherlands, Vol. 4, 1993, pp. 171-173.

[15] van Luijtelaar, E.L.J.M. and Coenen, A.M.L., Two types of electrocortical paroxysms in an inbred strain of rats, $\mathrm{Neu}$ rosci. Lett., 70 (1986) 393-397.

[16] Vergnes, M., Marescaux, C., Micheletti, G., Depaulis, A., Rumbach, L. and Warter, J.-M., Enhancement of spike and wave discharges by GABA mimetic drugs in rats with spontaneous petit mal-like epilepsy, Neurosci. Lett., 44 (1984) 91-94. 